\title{
A Rare Case of Paraneoplastic Encephalitis in Association With Breast Cancer
}

\author{
Kavita Agrawal ${ }^{\mathrm{a}, \mathrm{b}}$, Nirav Agrawal ${ }^{\mathrm{a}}$
}

\begin{abstract}
We present a case of a 47-year-old female who presented with diffuse dysesthetic pain in both legs for past 3 months. She described the sensation as of pins and needles. She also had balance difficulty and developed progressively worsening back pain and spasms. Relatives reported that the patient had memory difficulties. The patient would repeat questions even after the questions had already been answered. Magnetic resonance imaging (MRI) of cervical, thoracic and lumbosacral spine with and without contrast showed mild multilevel degenerative changes in cervical spine and minimal disc bulges from L1 to S1 levels with no areas of significant spinal canal or neural foraminal stenosis. MRI of brain was performed which showed abnormal T2/FLAIR hyperintensity along the left medial temporal lobe and additional small foci in the right centrum semiovale/corona radiate and bilateral periventricular white matter. Based on the MRI findings, a diagnosis of limbic encephalitis was made. A lumbar puncture was also done. Cerebrospinal fluid (CSF) showed 14 oligoclonal bands with elevated IgG synthesis. Paraneoplastic antibody panel showed high titers of amphiphysin antibody $(1: 15,360)$. Since this is a paraneoplastic antibody, computed tomography of chest, abdomen and pelvis was performed. It showed several enlarged right axillary lymph nodes. Ultrasound of right breast showed $4 \mathrm{~mm}$ hypoechoic nodule at 2 o'clock axis (upper inner quadrant). The biopsy of this lesion and axillary lymph node showed invasive ductal carcinoma of the breast. For further evaluation, MRI of bilateral breast was performed. It revealed additional areas of enhancements in upper outer and lower outer quadrants of right breast. Biopsy of the upper outer area of enhancement revealed invasive ductal carcinoma and ductal carcinoma in situ (DCIS). Biopsy of the lower outer aspect showed infiltrating lobular carcinoma and lobular carcinoma in situ (LCIS). Our report highlights a rare patient presenting with paraneoplastic limbic encephalitis with underlying breast cancer. When encountering a patient with unexplained neurologic symptoms, paraneoplastic neurological syndromes (PNSs) should be considered as part of the differential. And a thorough search of autoantibodies associated with PNS should be considered. If such autoantibodies are present, search for an underlying malignancy should be undertaken.
\end{abstract}

Manuscript submitted June 20, 2018, accepted July 20, 2018

aDepartment of Internal Medicine, Overlook Medical Center, 99 Beauvoir Avenue, Summit, NJ 07901, USA

${ }^{b}$ Corresponding Author: Kavita Agrawal, Department of Internal Medicine, Overlook Medical Center, 99 Beauvoir Avenue, Summit, NJ 07901, USA. Email: kaviagrawa189@gmail.com

doi: https://doi.org/10.14740/jmc3120w
Keywords: Encephalitis; Diffuse dysesthetic pain; Magnetic resonance imaging; Cerebrospinal fluid; Paraneoplastic neurological syndrome

\section{Introduction}

Paraneoplastic neurological syndromes (PNSs) are syndromes of nervous system disorder associated with cancer (that are not explained by other diagnoses). They are not caused by metastasis or direct tumor invasion of the nervous system. The spectrum of PNS includes paraneoplastic encephalomyelitis (PEM), paraneoplastic cerebellar degeneration (PCD), limbic encephalitis (found in our patient), opsoclonus myoclonus, subacute sensory neuronopathy and Lambert-Eaton myasthenic syndrome (LEMS). The neurologic dysfunction of PNS can precede ( $60 \%$ of cases) or follow ( $40 \%$ of cases) a diagnosis of cancer. The current hypothesis is that PNS is autoimmune in nature. An array of autoantibodies have been identified, some of which are associated with specific PNS and malignancies.

\section{Case Report}

A 47-year-old female presented with constant diffuse dysesthetic pain in both legs for the past 3 months. She described the pain as burning with pins and needles sensation. She also had progressively worsening back pain and spasms. It was associated with increasing balance difficulties. Initially, her symptoms were attributed to neuropathy (and worked up accordingly, Table 1). She was given a trial of amitriptyline and gabapentin with no noted improvement and her symptoms continued to worsen. A month after presentation, the patient's mother started noticing memory difficulties. The patient would repeat questions even after the questions had already been answered. She denied fever, chills, visual problems, headaches, rash, arthralgias, joint swelling, focal motor or sensory deficits, and bladder or bowel incontinence.

The patient had a past medical history of bacterial meningitis treated at age of 4 . She had gastric sleeve surgery 5 years ago. She had no known allergies. Her medications included vitamin B12 and vitamin D supplements. She denied smoking, alcohol use or illicit drug use. She denied any family history of autoimmune, rheumatologic or demyelinating disease. 
Table 1. Laboratory Data

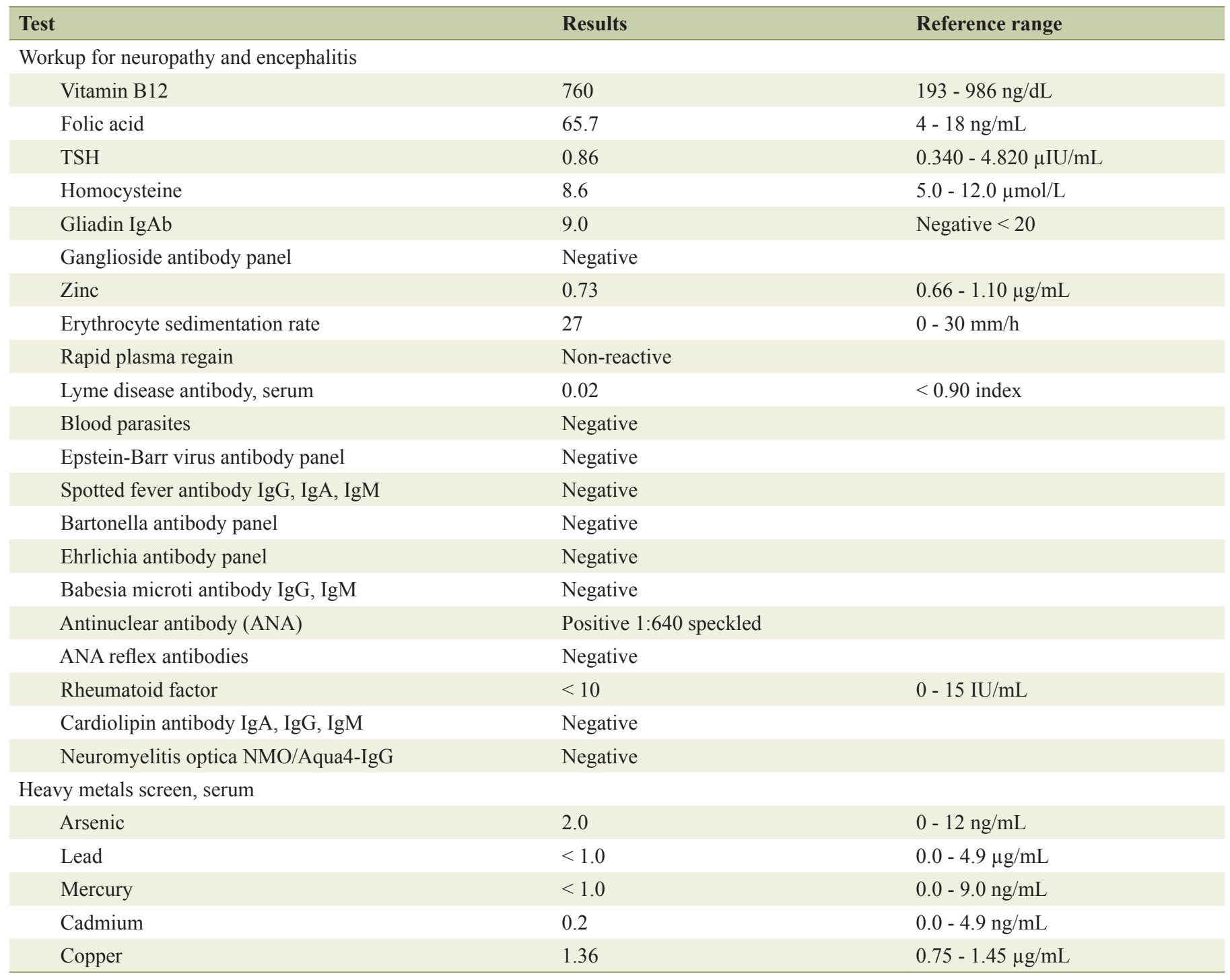

Physical exam revealed an overweight female with no apparent distress. Her pulse was 78 beats per minute, blood pressure was $111 / 70 \mathrm{~mm} \mathrm{Hg}$, respiratory rate was 16 breaths per minute, and oxygen saturation was $99 \%$ on room air. The speech was fluent without errors. On mini-mental state examination (MMSE), she recalled two out of three names at $15 \mathrm{~min}$ and had difficulty doing serial threes. Direct fundoscopic exam showed pink optic discs with sharp margins. Cranial nerves IXII were grossly intact. Tone and strength in all extremities was normal. Deep tendon reflexes were $1+$ and symmetric in bilateral upper extremities but unelicitable at bilateral knees and ankles. Sensory exam to soft and painful stimulus was normal in all four extremities. Her gait was very cautious and unsteady, although sufficiently strong to rise on heels and toes. Tandem walk was difficult. Romberg test was unsteady with both eyes opened and closed. Neck was supple with full range of motion. Lung, heart and abdomen exam was normal.

Magnetic resonance imaging (MRI) of cervical, thoracic and lumbosacral spine with and without contrast showed mild multilevel degenerative changes in cervical spine and minimal disc bulges from L1 to S1 levels with no areas of significant spinal canal or neural foraminal stenosis. MRI of brain was performed due to memory difficulties. It showed abnormal T2/ FLAIR hyperintensity along the left medial temporal lobe involving areas of hippocampus and amygdala, additional small foci in the right centrum semiovale/corona radiate and bilateral periventricular white matter (Fig. 1a). Based on the above findings, a diagnosis of limbic encephalitis was made. She also underwent extensive laboratory workup (Table 1) which was negative.

She underwent lumbar puncture. Cerebrospinal fluid (CSF) showed 44 white blood cells per microliter with $90 \%$ lymphocytes. Glucose and protein levels were within normal limits. CSF showed elevated oligoclonal bands and increased CNS IgG synthesis. Testing for PNS autoantibodies showed elevated anti-amphiphysin antibodies (1:15,360, Table 2). CSF 

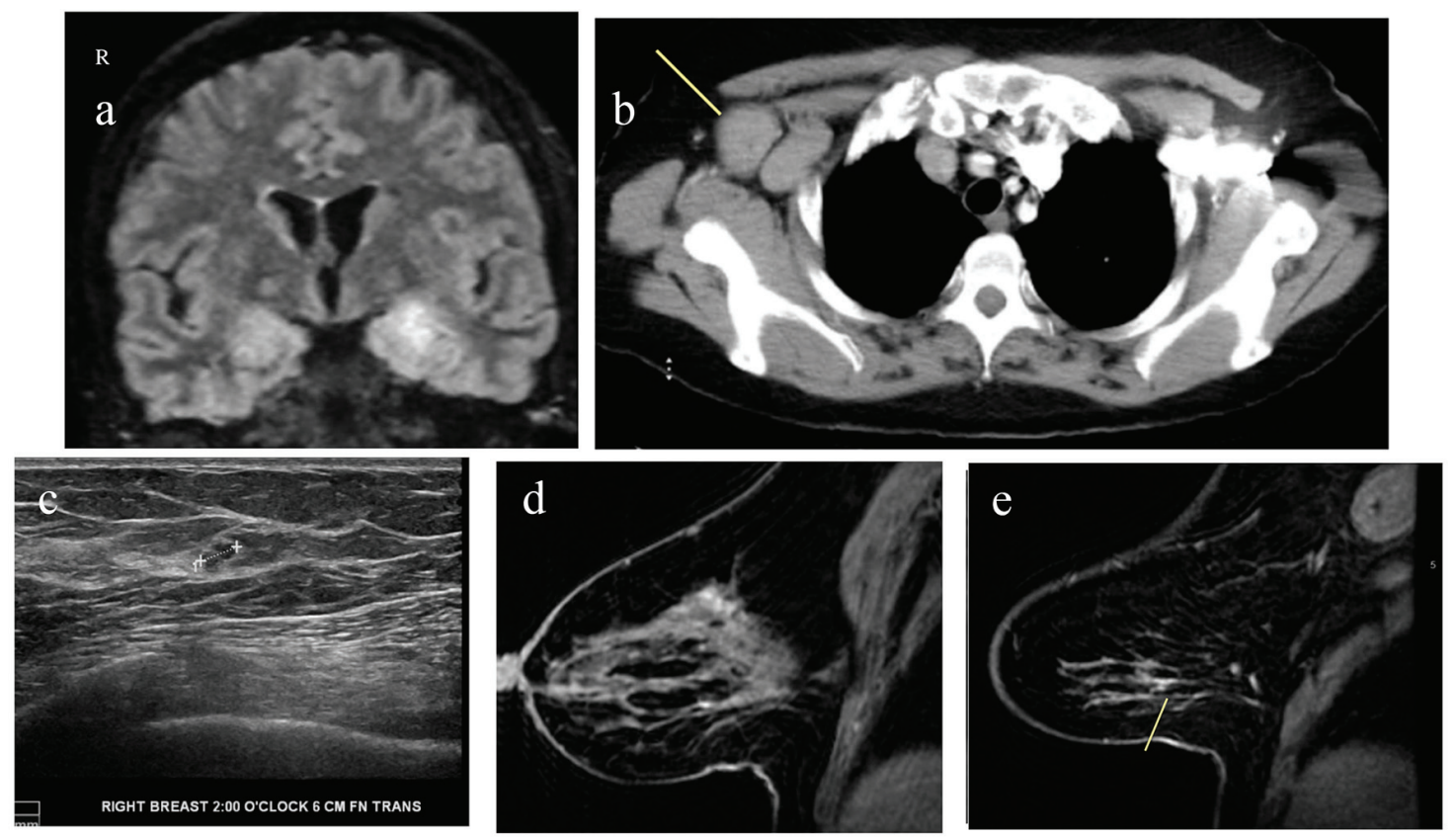

Figure 1. (a) MRI of brain with and without contrast showing T2/FLAIR hyperintensity along the medial temporal lobe involving portions of the hippocampus and amygdala. (b) CT of chest with contrast showing markedly enlarged axillary lymph nodes. (c) Ultrasound of right breast and axilla showing $4 \times 4 \times 3 \mathrm{~mm}$ hypoechoic lobulated nodule at 2 o'clock axis. (d) MRI of bilateral breast with and without contrast showing clumped enhancement in the upper outer quadrant of the right breast. (e) MRI of bilateral breast with and without contrast showing a small $8 \mathrm{~mm}$ area of patchy non-mass enhancement in the lower outer right breast.

meningitis/encephalitis panel was negative (Table 2). Since this is a paraneoplastic antibody, the patient underwent computed tomography (CT) of chest, abdomen and pelvis to rule out underlying malignancy. It showed right axillary lymphadenopathy (Fig. 1b). This raised concern for an underlying breast cancer that had spread to the axillary region. Ultrasound of right breast showed $4 \mathrm{~mm}$ hypoechoic nodule at 2 o'clock axis (Fig. 1c). The biopsy of this lesion and axillary lymph node showed invasive ductal carcinoma. For further evaluation, MRI of bilateral breast was performed. It revealed additional areas of enhancements in upper and lower outer quadrants of right breast (Fig. 1d, e). Biopsy of the upper outer area of enhancement revealed invasive ductal carcinoma and ductal carcinoma in situ (DCIS). Biopsy of the lower outer aspect showed infiltrating lobular carcinoma and lobular carcinoma in situ (LCIS). Our report highlights a rare case of PNS associated with breast cancer.

\section{Discussion}

PNSs are syndromes of nervous system disorder associated with cancer (that are not explained by other diagnoses). They are not caused by metastasis or direct tumor invasion of the nervous system $[1,2]$.

The current hypothesis regarding the pathogenesis of PNS is that they are immune mediated $[2,3]$. Our immune system generates antibodies or $\mathrm{T}$ cells response against tumor antigens. These antibodies or $\mathrm{T}$ cells cross-react with our normal neuronal tissues causing PNS. The theory is supported by CSF study in these patients. In one European study, lymphocytic pleocytosis was present in 39\%, elevated protein in $67 \%$ and oligoclonal bands of IgG in $63 \%$ [4]. Two of these findings were present in our patient's CSF.

Specific cancers have been associated with eliciting certain autoantibodies. These antibodies, in turn, are associated with dysfunction of specific parts of the nervous system. The symptoms of PNS vary based on the part of the nervous system affected. Symptoms can present acutely or sub-acutely and include tremors, visual complaints, ataxia, seizures, autonomic dysfunction, peripheral neuropathy and others (Table 3) [5]. Based on the symptoms, affected region of nervous system on imaging studies and types of autoantibodies elicited, the subtypes of PNS include PEM, PCD, limbic encephalitis (found in our patient), opsoclonus myoclonus, subacute sensory neuronopathy and LEMS [5].

PNS precedes $(60 \%$ of cases) or follows cancer diagnosis (40\% of cases) [2]. In a few instances, cancer cannot be found in a patient whose clinical syndrome is otherwise consistent with PNS. Such patients need to be on surveillance if cancer was not found on the initial workup. When encountering a patient with unexplained neurologic symptoms, PNS should be considered as part of the differential. A thorough search of autoantibodies associated with PNS should be considered (Table 4) [6, 7]. If such autoantibodies are present, search for an underlying malignancy should be undertaken.

The Purkinje cell antibody (PCA-1) was the first antibody described in relation to PNS in 1893 by Greenlee and Brashear. It was found in two patients with ovarian carcinoma and paraneoplastic cerebellar degeneration [8]. In our discussion, we 
Table 2. Cerebrospinal Fluid Analysis

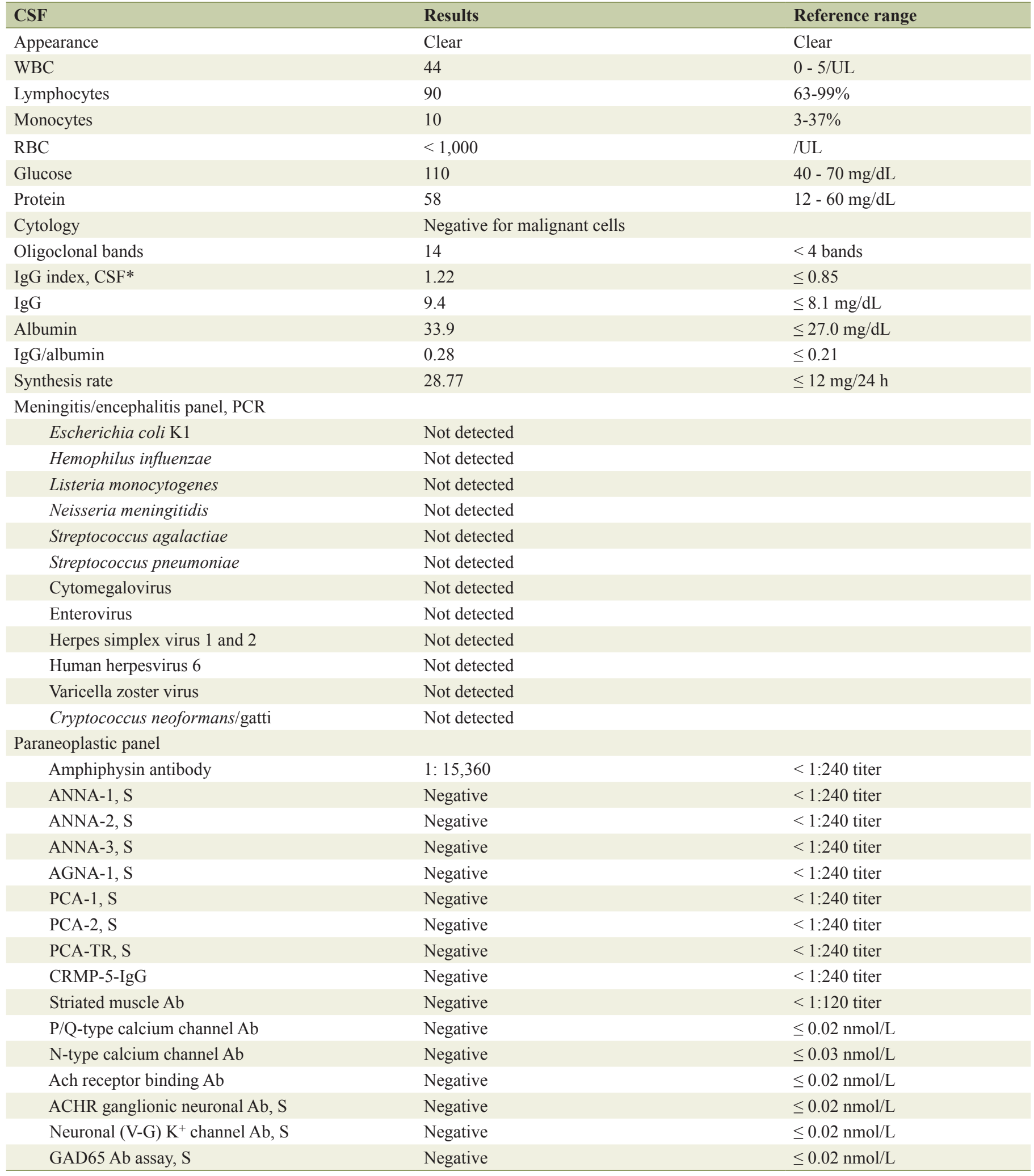

${ }^{*}$ CSF IgG index: CSF IgG to CSF albumin ratio compared to the serum IgG to serum albumin ratio. An increase in the index is an indicator of IgG production in the central nervous system. In our patient serum albumin $3.5 \mathrm{~g} / \mathrm{dL}$ and serum $\mathrm{ggG} 814 \mathrm{mg} / \mathrm{dL}$ (Reference: https://www.mayomedicallaboratories.com/test-catalog/Clinical+and+Interpretive/8271) 
Table 3. Clinical Features and Investigations Associated With Various PNS [5]

\begin{tabular}{|c|c|c|}
\hline Syndrome & Clinical feature & Investigations \\
\hline $\begin{array}{l}\text { Paraneoplastic } \\
\text { encephalomyelitis (PEM) }\end{array}$ & $\begin{array}{l}\text { Subacute involvement of more than one area of the CNS } \\
\text { includes cortical, limbic or brainstem encephalitis, } \\
\text { cerebellar dysfunction, myelitis }\end{array}$ & $\begin{array}{l}\text { MRI of the relevant part. CSF - pleocytosis, } \\
\text { elevated protein and oligoclonal bands }\end{array}$ \\
\hline Limbic encephalitis (LE) & Memory problems, seizures, mood and sleep abnormalities & $\begin{array}{l}\text { MRI brain T2/FLAIR hyperintensity involving } \\
\text { limbic structure. Abnormal CSF as above. } \\
\text { EEG epileptiform abnormality/focal slowing }\end{array}$ \\
\hline $\begin{array}{l}\text { Chronic gastrointestinal } \\
\text { pseudoobstruction }\end{array}$ & $\begin{array}{l}\text { Subacute progressive nausea, vomiting, abdominal } \\
\text { distention, pain and constipation }\end{array}$ & $\begin{array}{l}\text { GI motility study and autonomic reflex screen, } \\
\text { thermoregulatory sweat test for associated } \\
\text { autonomic dysfunction }\end{array}$ \\
\hline Opsoclonus myoclonus & $\begin{array}{l}\text { Involuntary chaotic saccades in all directions of gaze, } \\
\text { associated with myoclonus and ataxia frequently }\end{array}$ & $\begin{array}{l}\text { MRI brain usually normal. EMG } \\
\text { diagnosis of myoclonus }\end{array}$ \\
\hline $\begin{array}{l}\text { Subacute sensory } \\
\text { neuronopathy }\end{array}$ & $\begin{array}{l}\text { Numbness and pain onset in upper extremity, asymmetric. } \\
\text { Progression in less than } 12 \text { weeks }\end{array}$ & $\begin{array}{l}\text { Nerve conduction studies (NCS) - absent or } \\
\text { reduced SNAPs, MRI spine enhancing nerve } \\
\text { roots. Abnormal CSF as mentioned above. }\end{array}$ \\
\hline $\begin{array}{l}\text { Lambert-Eaton myasthenic } \\
\text { syndrome (LEMS) }\end{array}$ & $\begin{array}{l}\text { Proximal weakness with ocular and bulbar involvement. } \\
\text { Hypoactive deep tendon reflexes and mild dysautonomia } \\
\text { helps clinical differentiation from myasthenia gravis }\end{array}$ & $\begin{array}{l}\text { EMG-incremental response on repetitive } \\
\text { stimulation }\end{array}$ \\
\hline
\end{tabular}

will focus on antiamphiphysin antibodies (AAs) which were found in our patient. AAs are autoantibodies that react with $128 \mathrm{kD}$ protein amphiphysin I found in synaptic vesicles [9]. They were first reported in patients with Stiff-man syndrome and breast cancer [10]. More studies have shown that they can also be found in other PNS and malignancies. In 1999, Antoine et al performed testing to detect autoantibodies on 2,800 serum samples from patients with paraneoplastic syndromes. Of the five patients with AA, three had small cell lung cancer (one was diagnosed with cancer after 24 months of follow-up), one had breast cancer and one had ovarian cancer. In this group, PNS included sensory neuronopathy, limbic encephalitis, encephalomyelitis and LEMS [10].

The diagnosis of PNS is challenging especially in cases where a malignancy may not be initially apparent. However, in a patient with a consistent neurologic syndrome (as described above), PNS should be considered if no alternate diagnosis is apparent. The presence of personal or family history of cancer or autoimmune diseases provides clues to the diagnosis of PNS. On CSF analysis, presence of lymphocytic pleocytosis, increased protein or oligoclonal IgG are consistent with PNS. Depending on the syndrome, MRI of brain/spine will reveal T2/FLAIR hyperintensity in limbic structures, cerebellar atrophy or enhancing nerve roots. Depending on the syndrome, electromyography (EMG) will reveal findings such as incremental response on repetitive stimulation or myoclonus. Positive autoantibodies associated with PNS also make this diagnosis more likely. Therefore, a combination of clinical features, laboratory and imaging findings can help with the diagnosis of PNS (Tables 3 and 4) [5-7].
Our patient presented with pain in both legs and back associated with short term memory deficits. CSF analysis showed lymphocytic pleocytosis, oligoclonal bands, elevated IgG protein and positive AAs. MRI of brain showed hyperintensity along the left medial temporal lobe involving limbic structures. This led to a diagnosis of limbic encephalitis. A search was initiated to find out underlying malignancy with imaging. She was found to have right axillary lymphadenopathy and hypodensity in the right breast. This was subsequently proved as right breast cancer on biopsy. Patient underwent right mastectomy and was started on chemotherapy. This report illustrates a rare case of paraneoplastic limbic encephalitis associated with breast cancer.

\section{Conclusion}

Our report illustrates a rare case of a 47-year-old female who presented with neurological symptoms and diagnosed with paraneoplastic limbic encephalitis associated with breast cancer. A good clinical judgment based on different clinical, imaging and laboratory findings (as described above) can lead to early diagnosis of PNS and underlying malignancy. This is very important for prompt treatment to prevent irreversible neurological damage.

\section{Conflict of Interest}

The authors declare that there is no conflict of interest regard- 
Table 4. Paraneoplastic Antibodies Associated With Cancers and PNS [6, 7]

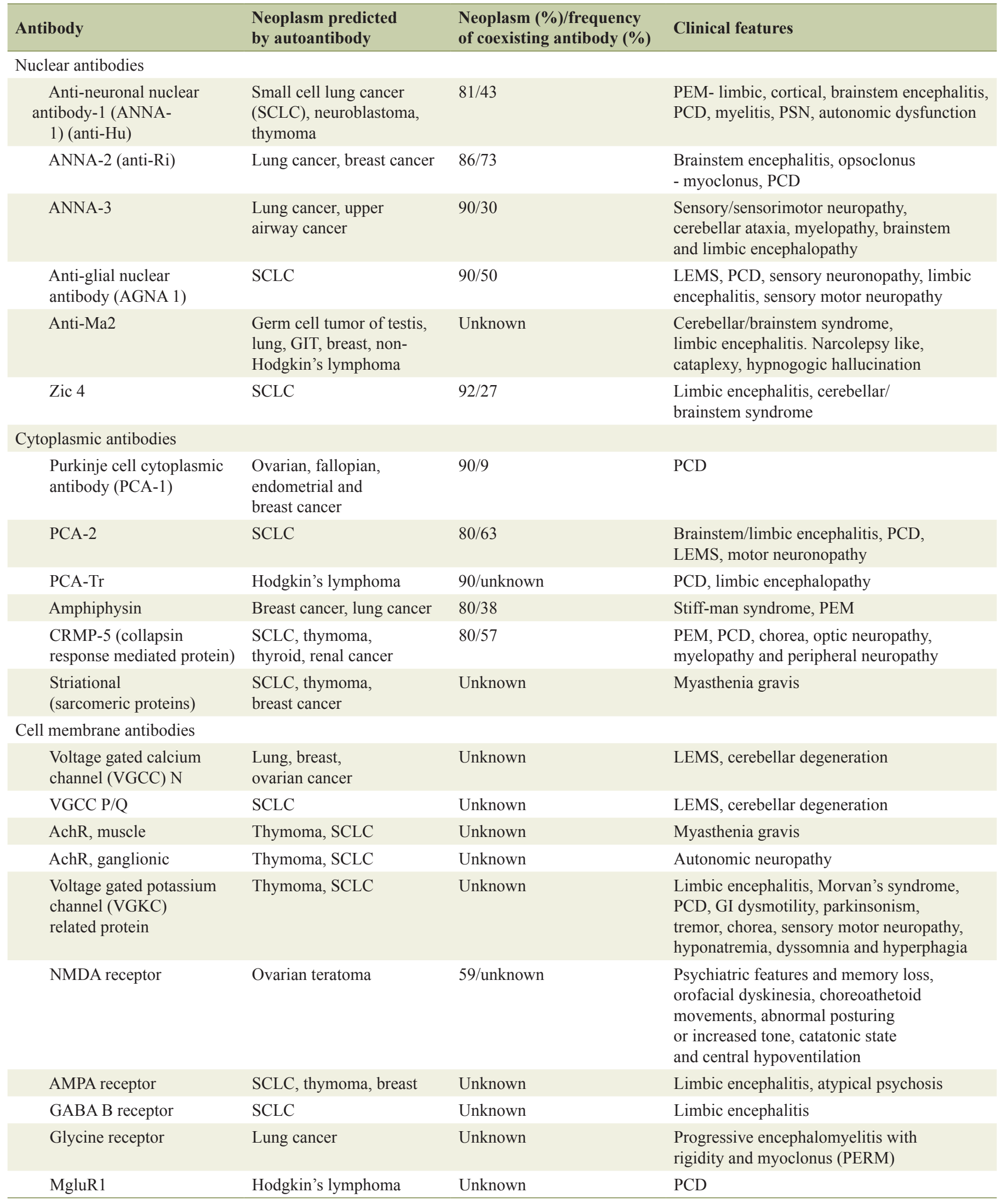


ing the publication of this paper.

\section{References}

1. Kannoth S. Paraneoplastic neurologic syndrome: A practical approach. Ann Indian Acad Neurol. 2012;15(1):612.

2. Josep Dalmau. Paraneoplastic neurologic syndromes. https://rarediseases.org/rare-diseases/paraneoplastic-neurologic-syndromes.

3. Dalmau J, Rosenfeild MR. Paraneoplastic Neurologic syndromes. In: Fauci AS, Braunwald EB, Kasper DL, et al., editors. Harrison's Principles of Internal Medicine. 17th ed. Vol. 1. New York: McGraw Hill Medical; 2008. pp. 623-627.

4. Psimaras D, Carpentier AF, Rossi C, Euronetwork PNS. Cerebrospinal fluid study in paraneoplastic syndromes. J Neurol Neurosurg Psychiatry. 2010;81(1):42-45.

5. Gozzard P, Maddison P. Republished: Which antibody and which cancer in which paraneoplastic syndromes?
Postgrad Med J. 2011;87(1023):60-70.

6. Lachance DH, Lennon VA. Paraneoplastic neurological autoimmunity In Neuroimmunology in Clinical Practice. In: Kalman B, Brannagan TH 3rd, editors. Oxford: Blackwell Publishing; 2008. pp. 210-217.

7. Rosenfeld MR, Dalmau J. Update on paraneoplastic and autoimmune disorders of the central nervous system. Semin Neurol. 2010;30(3):320-331.

8. Greenlee JE, Brashear HR. Antibodies to cerebellar Purkinje cells in patients with paraneoplastic cerebellar degeneration and ovarian carcinoma. Ann Neurol. 1983;14(6):609-613.

9. Saiz A, Dalmau J, Butler MH, Chen Q, Delattre JY, De Camilli P, Graus F. Anti-amphiphysin I antibodies in patients with paraneoplastic neurological disorders associated with small cell lung carcinoma. J Neurol Neurosurg Psychiatry. 1999;66(2):214-217.

10. Antoine JC, Absi L, Honnorat J, Boulesteix JM, de Brouker T, Vial C, Butler M, et al. Antiamphiphysin antibodies are associated with various paraneoplastic neurological syndromes and tumors. Arch Neurol. 1999;56(2):172-177. 